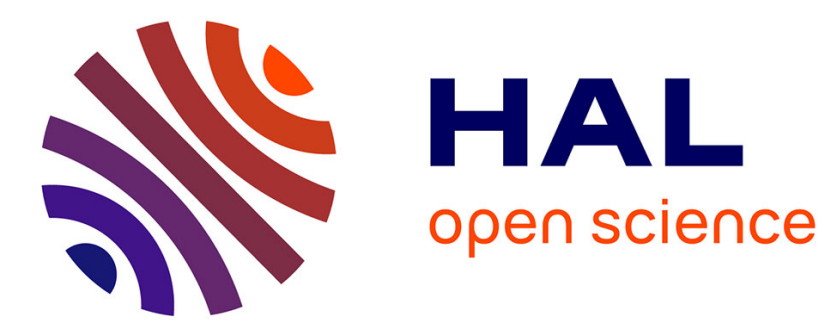

\title{
Bulk and surface vibrational and thermodynamical properties of fcc transition and noble metals : a systematic study by the continued fraction technique
}

G. Treglia, M.-C. Desjonquères

\section{> To cite this version:}

G. Treglia, M.-C. Desjonquères. Bulk and surface vibrational and thermodynamical properties of fcc transition and noble metals: a systematic study by the continued fraction technique. Journal de Physique, 1985, 46 (6), pp.987-1000. 10.1051/jphys:01985004606098700 . jpa-00210046

\section{HAL Id: jpa-00210046 https://hal.science/jpa-00210046}

Submitted on 1 Jan 1985

HAL is a multi-disciplinary open access archive for the deposit and dissemination of scientific research documents, whether they are published or not. The documents may come from teaching and research institutions in France or abroad, or from public or private research centers.
L'archive ouverte pluridisciplinaire HAL, est destinée au dépôt et à la diffusion de documents scientifiques de niveau recherche, publiés ou non, émanant des établissements d'enseignement et de recherche français ou étrangers, des laboratoires publics ou privés. 
Classification

Physics Abstracts

$65.50-68.30-68.40$

\title{
Bulk and surface vibrational and thermodynamical properties of fec transition and noble metals : a systematic study by the continued fraction technique
}

\author{
G. Tréglia $\left({ }^{*}\right)$ and M.-C. Desjonquères $\left({ }^{* *}\right)$ \\ (*) Laboratoire de Physique des Solides, Université Paris-Sud, Bât. 510, Centre d’Orsay, 91405 Orsay, France \\ ${ }^{* *}$ ) Service de Physique des Atomes et des Surfaces, Centre d'Etudes Nucléaires de Saclay, 91191 Gif sur Yvette, \\ France
}

(Reçu le 23 novembre 1984, révisé le 30 janvier 1985, accepté le 4 février 1985)

\begin{abstract}
Résumé. - Nous présentons une étude systématique des propriétés vibrationnelles et thermodynamiques des métaux nobles et de transition de structure cfc, dans le cadre d'un modèle comportant à la fois des forces centrales et des forces angulaires. Nous calculons les spectres de fréquence de phonons par une technique de fraction continue et nous en déduisons la contribution des phonons à diverses grandeurs thermodynamiques : chaleur spécifique à volume constant, entropie, déplacement carré moyen. Dans le volume, nous reproduisons bien l'ensemble des tendances expérimentales, à la fois qualitativement et quantitativement. Nous étudions ensuite les trois faces de bas indice de ces métaux. Leurs spectres sont fortement déformés par la présence de phonons de surface. Nous dégageons certaines tendances générales de l'étude de la variation due à la surface des grandeurs thermodynamiques. Par exemple, la variation de chaleur spécifique est maximale pour une température qui est une fraction constante $(\sim 0,175)$ de la température de Debye de volume. D'autre part, la composante parallèle du déplacement carré moyen dépend beaucoup plus de l'orientation de la surface que sa composante perpendiculaire.
\end{abstract}

\begin{abstract}
We present a systematic study of vibrational and thermodynamical properties of fcc transition and noble metals, using a model including central and angle-bending forces. The phonon frequency spectra are computed by means of the continued fraction technique; their knowledge enables us to calculate the contribution of phonons to various thermodynamical quantities such as the specific heat at constant volume, the entropy and the mean square displacements. For the bulk, our calculations reproduce all the experimental trends both qualitatively and quantitatively. Then we give results for the three low index faces of these metals. The surface spectra exhibit drastic deformations due to the existence of surface phonons. The variation of thermodynamical quantities due to the surface reveals some general trends. For example, the variation of specific heat is maximum at a temperature which is a constant fraction $(\sim 0.175)$ of the bulk Debye temperature. On the other hand, the lateral mean square displacement is much more dependent on the surface density of atoms than the perpendicular one.
\end{abstract}

\section{Introduction.}

The calculation of vibrational properties of crystals is of considerable interest for several reasons [1,2]. First, the phonon spectra can be measured by means of inelastic neutron scattering, infrared Raman or electron energy loss spectroscopy (EELS) experiments, and give informations on binding forces between atoms. Then, from the knowledge of the phonon frequency spectrum, one can derive all thermodynamical quantities of interest : vibrational entropy (required to study phase transformations as a function of temperature), specific heat, mean square displacement or equivalently the Debye temperature which is the essential ingredient in the Debye-Waller factor necessary for the interpretation of many experiments such as low energy electron diffraction (LEED), Mössbauer effect... Finally, if one assumes that the Lindemann criterion can be applied without change to any element, these mean square displacements can be used to evaluate melting temperatures.

However, even though the lattice dynamics of crystals has been an active topic of theoretical study during the past twenty years, most of the calculations have been performed in the low or high temperature limits [3-7]. Actually, the study of the intermediate temperature range requires tedious summations in $k$-space, which explains that, even for a given element, no complete investigation can be found, at least to our knowledge. Note that this difficulty increases for 
surfaces, due to the broken periodicity in one direction. Then, whereas the electronic properties of transition metals for example can be simply related to their position in a transition series, no equivalent systematic study exists for vibrational and thermodynamical properties (even though force constants are obviously related to electronic structure). On the other hand, it has long been known that an analogy can be made between the equation of motion of atoms in a crystal in the harmonic approximation and the Schrödinger equation for the crystal electronic states in the tight binding approximation [8]. As a consequence, the continued fraction technique, which avoids the summation in $k$-space and has been successfully used for systematic calculations of electronic properties of different solids, could be applied with the same purpose to study vibrational properties [9]. However, up to now, little attention has been paid to the possibility of using the continued fraction method in the latter case : this technique has only been worked out for some definite systems (Ni bulk and (001), (111) and (110) faces clean $[10,11]$ and with $[12,13]$ adsorbed hydrogen; W [14] and $\mathrm{Rh}$ [15] bulk and (001) face; $\mathrm{ZnS}$ [16, Ge, GaAs [17] bulk, Al coincidence boundaries [18]...) often to interpret particular experimental data.

The purpose of this paper is to start a systematic study of vibrational and thermodynamical properties, limiting ourselves in a first step to transition and noble metals which crystallize with the fcc structure. In section 2 we will first recall the characteristics of the lattice dynamical model we use (which includes central and angle bending forces) and discuss the determination of the force constant parameters; then we will show how the continued fraction technique can be applied to calculate the phonon frequency spectrum $n(v)$. In section 3 , we will present our results for the bulk phonon frequency spectra and thermodynamical properties of the eight fcc transition and noble metals : $\mathrm{Ni}, \mathrm{Cu}$; Rh, Pd, Ag; Ir, Pt, Au. These results will be successfully compared with the available experimental data and trends will be drawn. Finally, in section 4, a similar study will be performed for the three simple faces - (111), (100) and (110) - of these metals showing that, even when force constants do not vary at the surface, the breaking of some bonds induces drastic modifications of the phonon density projected on the surface and of the thermodynamical quantities.

\section{General formalism.}

We employ the same rotationally invariant lattice dynamical model as Castiel et al. [19], and Black et al. [11]. We assume central forces between first $(n=1)$ and second $(n=2)$ nearest neighbours and, in addition, angle bending interactions involving triplets of such neighbours.

Let us first consider the central part of the potential. It can be expanded around the equilibrium distances $R_{1}\left(R_{2}\right)$ between (next) nearest neighbours, this expansion being limited to second order in the harmonic approximation :

$$
V_{\mathrm{c}}(R) \simeq V_{\mathrm{c}}^{0}+\sum_{\substack{\text { (next) nearest } \\ \text { neighbour bonds }}}\left(\alpha_{n}\left(R-R_{n}\right)+\frac{\beta_{n}}{2}\left(R-R_{n}\right)^{2}\right)
$$

where $\alpha_{n}$ and $\beta_{n}(n=1,2)$ are the central force constants and $V_{c}^{0}$ the static central potential. With these definitions, the force acting on a given atom (labelled 0 ) due to its displacement $\mathbf{u}_{0}$ and that of a $n$-neighbouring atom at site $i$ (and therefore at a distance $R_{0 i}=R_{n}$ ) $\mathbf{u}_{i}$ is given by :

$$
F_{0 \lambda}^{\{0, i\}}=\frac{\alpha_{n}}{R_{n}} R_{0 i}^{\lambda}+\frac{\alpha_{n}}{R_{n}} u_{0 i}^{\lambda}+\left(\beta_{n}-\frac{\alpha_{n}}{R_{n}}\right)\left(\frac{\mathbf{u}_{0 i} \cdot \mathbf{R}_{0 i}}{R_{n}^{2}}\right) R_{0 i}^{\lambda}
$$

where $\mathbf{u}_{0 i}=\mathbf{u}_{i}-\mathbf{u}_{0}$ and $R_{0 i}^{\lambda}\left(u_{0 i}^{\lambda}\right)$ is the $\lambda^{\text {th }}$ component of $\mathbf{R}_{0 i}\left(\mathbf{u}_{0 i}\right)$ in the $\lambda$ direction. In practice, we will replace in the following the parameters $\alpha_{n}$ by $\lambda_{n}=\alpha_{n} / R_{n}$. These parameters are not independent since, due to the bulk equilibrium condition, they satisfy the relation : $\lambda_{1}=-\lambda_{2}[17]$.

Let us now discuss the angular part of the potential in more detail. As in the case of central forces, we expand this part around an equilibrium angle $\theta_{i \hat{0} j}$ between two first or second nearest neighbour bonds $\{0, i\},\{0, j\}$ where $i$ and $j$ are also first or second nearest neighbours.

$$
V_{\mathrm{a}}(\theta) \simeq V_{\mathrm{a}}^{0}+\sum_{i \hat{0}_{j}} \gamma_{i 0 j}\left(\cos \theta-\cos \theta_{i \hat{0} j}\right)+\sum_{i \hat{0} j} \frac{\delta_{i 0 j}}{2}\left(\cos \theta-\cdot \cos \theta_{i \hat{0} j}\right)^{2}
$$

where $V_{\mathrm{a}}^{0}$ is the static angular potential.

The variation of cosines is then calculated up to second order in the displacements $\mathbf{u}_{0 i}, \mathbf{u}_{i j}$ and $\mathbf{u}_{0 j}$. The force acting on atom 0 due to the angular distortion of the triangle $\{0, i, j\}$, in direction $\lambda$, is obtained by derivating the potential with respect to $u_{0}^{\lambda}$ :

$$
\begin{aligned}
F_{0 \lambda}^{\{0, i, j\}}=\sum_{\mu=1,3}\left\{\left(Q_{0 i} \delta^{\lambda \mu}+P_{0 i}^{\lambda \mu}\right) u_{0 i}^{\mu}+\left(Q_{0 j} \delta^{\lambda \mu}+P_{0 j}^{\lambda \mu}\right) u_{0 j}^{\mu}+\right. & \\
& \left.+\left(Q_{i j} \delta^{\lambda \mu}+P_{i j}^{\lambda \mu}\right) u_{i j}^{\mu}\right\}+Q_{0 i} R_{0 i}^{\lambda}+Q_{0 j} R_{0 j}^{\lambda}+Q_{i j} R_{i j}^{\lambda}
\end{aligned}
$$


where $P$ and $Q$ are functions of the angles of the triangle $\{0, i, j\}$ and of the direction cosines $d_{0 i}^{\lambda}, d_{0 j}^{\lambda}, d_{i j}^{\lambda}(\lambda=1$, $2,3)$ of $\mathbf{R}_{0 i}, \mathbf{R}_{0 j}, \mathbf{R}_{i j}$, the expressions of which are given in the appendix.

The equation of motion for atom 0 , with mass $m$, is :

$$
m \frac{\mathrm{d}^{2} u_{0}^{\lambda}(t)}{\mathrm{d} t^{2}}=F_{0 \lambda}=\sum_{\{0, i\}} F_{0 \lambda}^{\{0, i\}}+\sum_{\{0, i, j\}} F_{0 \lambda}^{\{0, i, j\}}
$$

Writing $u_{0}^{\lambda}(t)$ as $\left\{u_{0}^{\lambda} \mathrm{e}^{-2 i \pi v t}\right\}$ and taking into account that the total force vanishes when there are no displacements, equation (5) becomes :

$$
v^{2} u_{0}^{\lambda}=-\frac{F_{0 \lambda}}{4 \pi^{2} m}=\sum_{i, \mu} \mathbb{D}_{0 i}^{\lambda \mu} u_{i}^{\mu}
$$

where $\mathbb{D}$ is the dynamical matrix and $i$ is a first or second neighbour of atom 0 . This new equation of motion is formally identical with the Schrödinger equation for the eigenstates of electrons in the tightbinding approximation : the eigenenergy, wave function and Hamiltonian matrix elements are replaced by the square of frequency $\left(v^{2}\right)$, the displacements $\left(u_{i}^{\mu}\right)$ and the dynamical matrix elements $\mathbb{D}_{0 i}^{\lambda \mu}$ respectively. As a consequence, one can define a Green operator $G\left(v^{2}\right)=\left(v^{2} \mathbb{D}-\mathbb{D}\right)^{-1}$, the diagonal matrix elements of which can be calculated using the continued fraction technique $[20,21]$

$$
G_{00}^{\lambda \lambda}\left(v^{2}\right)=\frac{1}{\sqrt{\nu^{2}-a_{1}^{\lambda}}}-\frac{b_{1}^{\lambda}}{v^{2}-a_{2}^{\lambda}}-\cdots-\frac{b_{n}^{\lambda}}{\sqrt{v^{2}-a_{n+1}^{\lambda}}} \ldots
$$

From this Green function we can deduce the amplitude weighted phonon density of states along a direction $\lambda$ at site 0 :

$$
n_{0}^{\lambda}(v)=-\frac{\operatorname{Im}}{\pi} \lim _{\varepsilon \rightarrow 0^{+}}\left(2 v G_{00}^{\lambda \lambda}\left(v^{2}+i \varepsilon\right)\right)
$$

(which will be referred to as $\lambda$-spectral density in the following) and then the total density of states :

$$
n_{0}(v)=\frac{1}{3} \sum_{\lambda} n_{0}^{\lambda}(v)
$$

(in the bulk, $n_{0}(v)$ is site-independent and is then denoted $n(v)$ ). We use the recursion method [20] to calculate the coefficients $a_{n}$ and $b_{n}$ of the continued fraction (Eq. (8)). In practice, only a finite number $N$ of exact coefficients can be computed but, due to their fast convergence in the absence of band gaps, they are replaced by their asymptotic values (which are related to the band edges) beyond the $N^{\text {th }}$ level $\left(a_{n>N}=a_{\infty}, b_{n>N}=b_{\infty}\right)$ which provides us with a termination for the continued fraction. It is straightforward to make the standard computer programs elaborated for electronic calculations suitable for phonon ones. The main change comes from replacing Slater Koster matrices by dynamical ones. The crystal field integrals at site 0 are replaced by the $(3 \times 3)$ matrix $\mathbb{D}_{00}$ with elements.

$$
\mathbb{D}_{00}^{\lambda \mu}=\frac{1}{4 \pi^{2} m}\left(\sum_{\{0, i\}}\left\{\lambda_{i} \delta^{\lambda \mu}+\left(\beta_{i}-\lambda_{i}\right) d_{0 i}^{\lambda} d_{0 i}^{\mu}\right\}+\sum_{\{0, i, j\}}\left\{\left(Q_{0 i}+Q_{0 j}\right) \delta^{\lambda \mu}+P_{0 i}^{\lambda \mu}+P_{0 j}^{\lambda \mu}\right\}\right)
$$

and the hopping integrals between site 0 and $i$ by the $(3 \times 3)$ matrix $\mathbb{D}_{0 i}$ :

$$
\mathbb{D}_{0 i}^{\lambda \mu}=\frac{1}{4 \pi^{2} m}\left(-\lambda_{i} \delta^{\lambda \mu}-\left(\beta_{i}-\lambda_{i}\right) d_{0 i}^{\lambda} d_{0 i}^{\mu}+\sum_{\{0, j\}}\left\{\left(Q_{i j}-Q_{0 i}\right) \delta^{\lambda \mu}+P_{i j}^{\lambda \mu}-P_{0 i}^{\lambda \mu}\right\}\right)
$$

where $\lambda_{i}\left(\beta_{i}\right)$ stands for $\lambda_{1}, \lambda_{2}\left(\beta_{1}, \beta_{2}\right)$ depending on the nature of the $\{0, i\}$ bonding (first or second neighbours); for the expression of $P$ and $Q$, see the appendix.

From the knowledge of $n_{0}(v)$, we can compute the contribution of phonons to thermodynamical properties. The specific heat at constant volume per atom $C_{\mathrm{v}}$ is given by :

$$
C_{\mathrm{v}}=3 k_{\mathrm{B}} \int_{0}^{v_{\max }} n_{0}(v)\left(\frac{h v}{2 k_{\mathrm{B}} T}\right)^{2}\left(\operatorname{coth}^{2}\left(\frac{h v}{2 k_{\mathrm{B}} T}\right)-1\right) \mathrm{d} v
$$


where $k_{\mathrm{B}}$ and $h$ are the Boltzmann and Planck constants respectively, $T$ the absolute temperature and $v_{\max }$ the maximum phonon frequency. The product of the entropy by temperature (entropic energy), which is necessary to compute the free energy, is written :

$$
T S=E-3 k_{\mathrm{B}} T \int_{0}^{v_{\max }} n_{0}(v) \ln \left(2 \sinh \left(\frac{h v}{2 k_{\mathrm{B}} T}\right)\right) \mathrm{d} v
$$

where $E$ is the energy :

$$
E=\frac{3 h}{2} \int_{0}^{v_{\max }} v n_{0}(v) \operatorname{coth}\left(\frac{h v}{2 k_{\mathrm{B}} T}\right) \mathrm{d} v
$$

Finally, the mean square displacement along the $\lambda$ direction can be calculated from the equation :

$$
\left\langle u_{\lambda}^{2}\right\rangle=\frac{h}{8 \pi^{2} m} \int_{0}^{v_{\max }} \frac{n_{0}^{\lambda}(v)}{v} \operatorname{coth}\left(\frac{h v}{2 k_{\mathrm{B}} T}\right) \mathrm{d} v .
$$

Let us recall that these formulae have been mainly used before, in the low and high temperature limits for which the complete knowledge of $n_{0}(v)$ is not needed. The major advantage of the present continued fraction technique is that, $n_{0}(v)$ being known analytically, all the previous thermodynamical functions are easily calculated in the whole temperature range.

\section{Determination of the force constants of fcc metals.}

Let us first determine the exact number of parameters required for an accurate calculation of fcc crystal phonon spectra. The central forces can be written in terms of three independent parameters $\beta_{1}, \beta_{2}$ and $\lambda_{1}\left(\lambda_{2}=-\lambda_{1}\right)$. Due to the geometry of the fcc lattice, the only important contribution to angular forces comes from the distortion of equilateral triangles formed by a triplet $\{0, i, j\}$ of first nearest neighbours. Consequently, all the angles $\theta_{i \hat{0} j}, \theta_{i \hat{j} 0}$ and $\theta_{0 \hat{j}}$ involved in the calculation are equal $\left(\theta_{0}=60^{\circ}\right)$ and we are left with two parameters $\gamma, \delta$ only. In the present case, $\gamma$ and $\delta$ can be assimilated to the same constant $\delta$ as in the work of Castiel et al. [17]. Let us notice however that, due to their expansion of the non-central potential, our definition of $\delta$ differs from theirs (which is the same as that used by Black et al. [11]) by a factor of 3 . This factor is obtained by developing our expression (3) to second order in $\mathrm{d} \theta$ and identifying the coefficient of the second term with theirs :

$$
\begin{gathered}
\delta\left(\sin ^{2} \theta_{0}-\cos \theta_{0}\right)=\sin ^{2} \theta_{0} \delta^{\text {Castiel-Black }} \\
\delta=3 \delta^{\text {Castiel-Black }} .
\end{gathered}
$$

In summary, the equation of motion is expressed with the help of four independent force constants $\left(\beta_{1}, \beta_{2}, \lambda_{1}\right.$ and $\left.\delta\right)$ and then four equations are required.
By going to the continuous limit of the equation of motion [15] and comparing the results with the equations of elasticity theory, one can obtain the relations between the force constants and the elastic constants. This provides us with three equations. A fourth one has still to be found. A close examination of the fcc experimental phonon dispersion curves reveals that the largest frequencies at points $X$ and $L$ are almost equal (to less than $3 \%$ ) [22-27]. Then, the dispersion curves will be well fitted by a set of parameters ensuring the equality of these two frequencies : this gives us the fourth equation. Solving them, we obtain :

$$
\begin{aligned}
& \beta_{1}=\frac{a}{16}\left(C_{11}+10 C_{12}+4 C_{44}\right) \\
& \beta_{2}=\frac{a}{16}\left(3 C_{11}-2 C_{12}-4 C_{44}\right) \\
& \lambda_{1}=\frac{a}{48}\left(3 C_{11}-2 C_{12}-4 C_{44}\right) \\
& \frac{\delta}{a^{2}}=\frac{a}{6}\left(C_{44}-C_{12}\right)
\end{aligned}
$$

where $a$ is the lattice parameter.

The values of the elastic constants for the eight fcc transition and noble metals have been drawn from the compilation by Ducastelle [28]. They lead to force constants displayed in table I.

The maximum frequency is easily expressed in terms of these force constants :

$$
v_{\max }=v_{\max }^{\mathrm{L}}=v_{\max }^{\mathrm{X}}=\frac{1}{2 \pi} \sqrt{\frac{8}{m}\left(\beta_{1}+\lambda_{1}+2 \delta\right)}
$$

and can be compared with the experimental values. In table I, one can see that the agreement is satisfactory. Equation (17) also provides us with a convenient check of the consistency of our parameters since the asymptotic values of the continued fraction coefficients $a_{\infty}$ and $b_{\infty}$ are related to $v_{\max }$ by the simple formulae

$$
\begin{aligned}
& a_{\infty}=\frac{v_{\max }^{2}}{2} \\
& b_{\infty}=\frac{v_{\max }^{4}}{16}=\frac{a_{\infty}^{2}}{4} .
\end{aligned}
$$

Note that this check is more drastic than in the case of electrons since there is a relation between $a_{\infty}$ and $b_{\infty}$ coming from the constraint that the minimum frequency should be : $v_{\min }=0$.

Let us remark that our determination of force constants, which uses the continuum limit, is, in principle, more suited to the description of the low frequency part of the dispersion curves. Another procedure would have been to fit them in the whole range of frequencies. However, since the dispersion curves of Ir and $\mathrm{Rh}$ are not available, we have preferred 
Table I. - Force constants in units $10^{12} \mathrm{dyn} / \mathrm{cm}^{2}$; theoretical $\left(v_{\text {mix }}^{\text {th }}\right)$ and experimental $\left(v_{\max }^{\exp }\right)$ maximum phonon frequencies in units $\mathrm{THz}$; experimental melting points $(\mathrm{K})$ and experimental Debye temperatures $(\mathrm{K})$ in the low $\left(\theta_{0}^{\mathbf{B}}\right)$ and high $\left(295 \mathrm{~K}: \theta_{\infty}^{\mathbf{B}}\right)$ temperature limits. a is the lattice parameter.

\begin{tabular}{|c|c|c|c|c|c|c|c|c|c|}
\hline & & $\mathrm{Ni}$ & $\mathrm{Cu}$ & $\mathrm{Rh}$ & Pd & $\mathrm{Ag}$ & Ir & $\mathrm{Pt}$ & $\mathrm{Au}$ \\
\hline \multirow{5}{*}{ Theory } & $\beta_{1} / a$ & 1.39 & 1.065 & 2.005 & 1.42 & 0.775 & 2.525 & 1.97 & 1.3 \\
\hline & $\beta_{2} / a$ & -0.035 & -0.025 & 0.025 & 0.04 & 0 & 0.135 & 0.145 & 0.05 \\
\hline & $\lambda_{1} / a$ & -0.01 & -0.01 & 0.01 & 0.015 & 0 & 0.045 & 0.05 & 0.015 \\
\hline & $\delta / a^{3}$ & -0.01 & -0.025 & -0.07 & -0.06 & -0.025 & 0.01 & -0.095 & -0.07 \\
\hline & $v_{\max }^{\text {th }}$ & 9.7 & 7.9 & 8.4 & 7.0 & 5.3 & 8 & 5.9 & 4.7 \\
\hline \multirow{4}{*}{ Experiments } & $v_{\max }^{\exp }$ & $9[22]$ & $7.34[23]$ & & $7.00[24]$ & $5.00[25]$ & & $5.81[26]$ & $4.67[27]$ \\
\hline & $T_{\mathrm{m}}^{\exp }$ & 1725 & 1357 & 2239 & 1824 & 1234 & 2727 & 2043 & 1336 \\
\hline & $\theta_{0}^{\mathrm{B}}[29]$ & 450 & 343 & 480 & 274 & 225 & 420 & 240 & 165 \\
\hline & $\theta_{\infty}^{\mathrm{B}}[30]$ & 390 & 310 & 350 & 290 & 220 & 290 & 225 & 185 \\
\hline
\end{tabular}

the first method to treat the eight metals on the same basis. Moreover our force constants are in very good agreement with those obtained within the second method by Castiel et al. [19]. Furthermore, as mentioned above, our calculated $v_{\max }$ are in good agreement with experimental values.

Finally, let us notice that, instead of fitting our force constants, we could try to calculate them a priori from the electronic structure. Unfortunately, in our formalism, pair interactions play a leading rôle though it is known that the total energy cannot be written as a sum of such interactions [28]. However angular forces introduce interactions between triplets which could reconcile both approaches, so that one can hope that all force constants could be calculated a priori, provided that some progress is made in the knowledge of the repulsive contribution.

\section{Application to bulk fec metals.}

4. 1 BULK PHONON DENSITIES OF STATES. - The bulk phonon densities of states of the eight fcc transition metals are shown in figure 1 . The most striking feature is the similarity of their shape, which is then characteristic of the fcc structure. This general shape is in very good agreement with previous calculations involving summations in $k$-space as can be seen from the inset of figure 1 [23]. However, our curves present some oscillations which are absent from the exact one. They are due to our approximation for the termination of the continued fraction which forbids us to reproduce accurately Van Hove singularities even with a large number of exact coefficients (here $N=17$ levels, which requires a cluster of 28595 atoms). Nevertheless these oscillations lead to completely

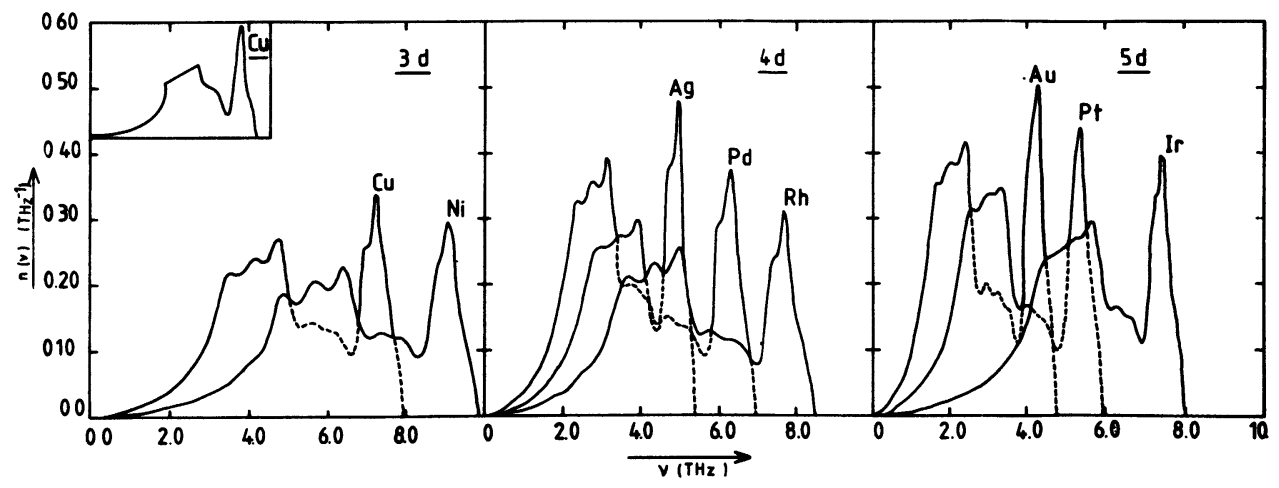

Fig. 1. - Bulk phonon densities of states of the fcc metals of the $3 \mathrm{~d}, 4 \mathrm{~d}$ and $5 \mathrm{~d}$ series. In the inset is exhibited the Cu density of states calculated using $k$-summation [23]. 
negligible errors on the thermodynamical quantities which are obtained by integrating over the frequencies.

The existence of a general shape for fcc phonon densities of states can be understood by noticing that one of the force constants $\left(\beta_{1}\right)$ prevails over the others by an order of magnitude (see Table I). However, using a model with only one force constant implies a universal curve when the frequencies are scaled by $v_{\max }$. This is not the case here, since the influence of noncentral forces lead to an additional increase of the density of states at low frequencies. In other words, if, on the low frequency side, the tail extends over a frequency range which is only a small fraction of $v_{\max }$, one can conclude that non-central forces are important. Using such a simple argument in figure 1, one can arrange the eight metals is ascending order with respect to the importance of angular forces; this gives :

\section{Ir, Ni, Cu, $\{\mathrm{Rh}, \mathrm{Pd}, \mathrm{Ag}\}, \mathrm{Pt}, \mathrm{Au}$}

which roughly corresponds to what could be guessed from table I.

Finally, one notes that $v_{\max }$ decreases along a transition series for fcc metals, as a consequence of the similar behaviour of the elastic constants [28].

\subsection{THERMODYNAMICAL PROPERTIES.}

4.2.1 Specific heat at constant volume. - The phonon contribution to the specific heat at constant volume (per atom) of the eight fcc metals has been calculated using formula [12] in the whole temperature range; the results are given in figure 2 . The eight curves have obviously the same well-known shape : they behave as $T^{3}$ at low temperature $(T<50 \mathrm{~K})$ and tend to the asymptotic value $3 k_{\mathrm{B}}$ when $T$ goes to infinity. The smaller the phonon spectrum width $\left(v_{\max }\right)$, the more rapidly the curves reach their limit. In order to compare these results with experiments in the low and intermediate temperature range, we have used the experimental values of $C_{\mathrm{p}}$ compilated by Hultgren

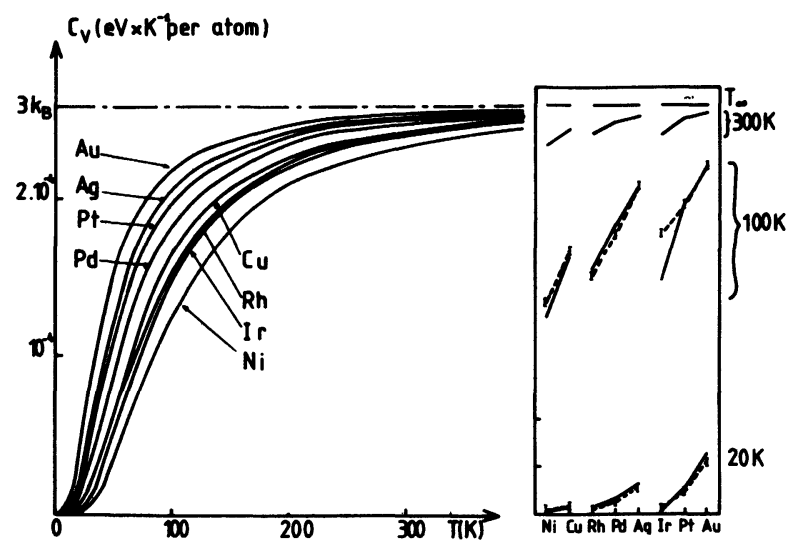

Fig. 2. - Variation of the phonon contribution $\left(C_{\mathrm{v}}\right)$ to the specific heat at constant volume as a function of the temperature. On the right hand side, we give the evolution of $C_{\mathrm{v}}$ along the transition series at given temperatures $(-$ : theory ; ----- experiments [31] : see text). et al. [31]. In this temperature range $(T<100 \mathrm{~K})$, the relative difference between $C_{\mathrm{p}}$ and $C_{\mathrm{v}}$ is rather small and can often be neglected. From these data we have subtracted the linear electronic contribution given by the same authors to obtain the experimental points of figure 2 . The experimental trends are well reproduced : $C_{\mathrm{v}}$ increases along a transition series and from the first transition series to the third one. Moreover, the calculated values are in quantitative agreement with experiments even in the intermediate temperature range $(100 \mathrm{~K})$ for which neither the low nor the high temperature developments are valid (see Fig. 2).

4.2.2 Vibrational entropy. - A similar systematic study has been performed for the phonon contribution to the entropic energy TS (Eq. (13)). The corresponding curves are drawn in figure 3 in the temperature range $\left(0, T_{\mathrm{m}}\right)\left(T_{\mathrm{m}}\right.$ : melting temperature). Here again, both limits are well reproduced : TS varies very slowly with $T$ at very low temperature $\left(\propto T^{4}\right)$ and diverges as $T \log T$ in the high temperature limit. This last limit provides us with a useful check of the validity of high temperature developments : one finds that these developments are justified above $600 \mathrm{~K}$. Let us remark that $C_{\mathrm{v}}$ has also reached its limit for $T>600 \mathrm{~K}$. As a consequence, the temperature range in which the full knowledge of $n(v)$ is necessary is : $50 \mathrm{~K} \lesssim T \lesssim 600 \mathrm{~K}$.

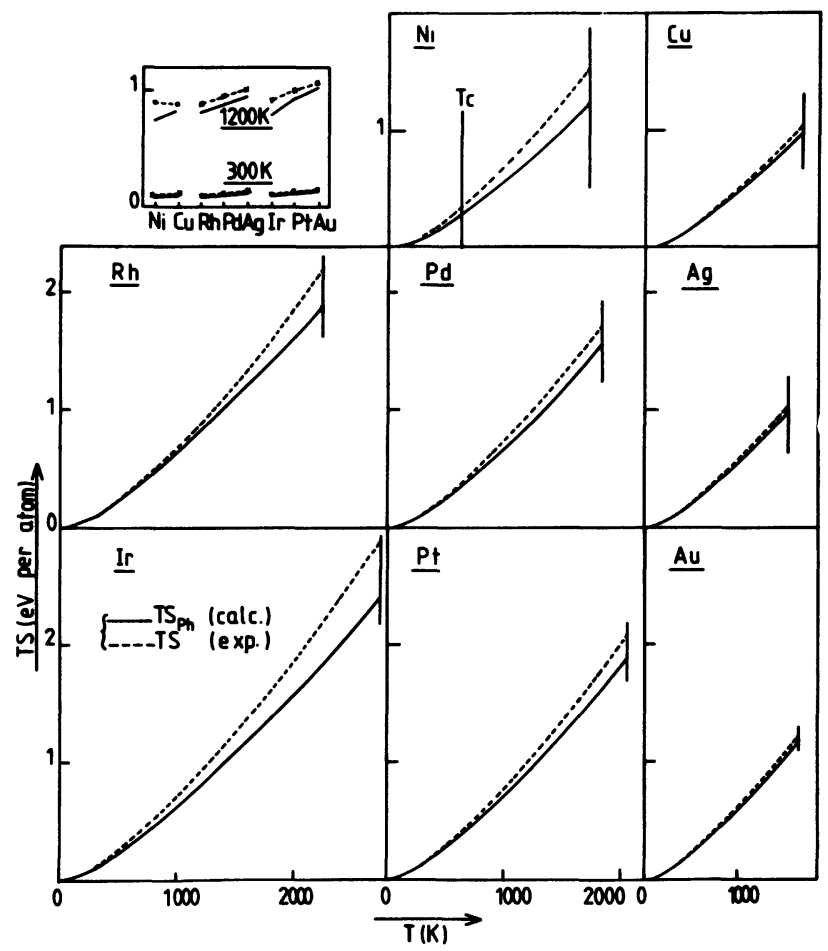

Fig. 3. - Variation of the phonon contribution to the entropic energy (-TS $-T S_{\mathrm{ph}}$ ) as a function of temperature up to the melting point, compared to the experimental total entropic energy (--- TS) [31] above room temperature. We have also indicated the Curie temperature of nickel $\left(T_{\mathrm{c}}\right)$. In the inset is shown the variation of $T S_{\mathrm{ph}}$ and $T S$ along the transition series at given temperatures. 
On the same figure 3 we have plotted the experimental values of $T S$ as a function of $T$, for $T>300 \mathrm{~K}$ [31]. The most striking feature is that the phonon contribution is largely predominant for any metal and at any temperature. The difference between the experimental total entropic energy and the calculated phonon contribution can be attributed to the electronic term (and possibly a magnetic one for $\mathrm{Ni}$ ) since this difference decreases along a transition series (see Fig. 3). Actually, the electronic contribution is proportional to $n\left(E_{\mathrm{F}}\right)$, which gets smaller for fcc at the end of a transition series, and becomes quasinegligible for noble metals since their $\mathrm{d}$ band is filled. Moreover, nearby the melting temperature, anharmonic effects may play a non-negligible rôle.

The variation of the phonon contribution to the entropic energy along a transition series at a given temperature $(300 \mathrm{~K}, 1200 \mathrm{~K})$ is displayed in the inset of figure 3. Similarly to $C_{\mathrm{v}}$, it increases rather slowly along a transition series and from the first to the third series.

In conclusion, the agreement between theory and experiments for $T \gtrsim 300 \mathrm{~K}$ in the case of entropy and $T \lesssim 300 \mathrm{~K}$ for the specific heat shows that our calculation method gives good results on the whole range of temperature.

4.2.3 Mean square displacement and Debye temperature. - We have calculated the mean square displacements $\left\langle u^{2}\right\rangle$ as a function of $T$ using equation (15). In figure 4 we show the resulting values of the ratio $\left(\sqrt{\left\langle u^{2}\right\rangle} / r_{0}\right)$ where $r_{0}$ is the interatomic distance. This ratio increases very slowly in the very low temperature range $(T<20 \mathrm{~K})$ and diverges as $\sqrt{T}$ at high temperature. For a given temperature outside the low temperature range, one finds that, if we arrange the eight metals in ascending order with respect to this ratio, the resulting sequence :

$$
\text { Ir, (Rh, Pt), Ni, Pd, (Cu, Au), Ag }
$$

is also that of decreasing cohesive energies.

It has long been known that a correlation can be established between melting temperatures and cohesive energies. It is then tempting to see to what extent a similar correlation can be made between $\left(\sqrt{\left\langle u^{2}\right\rangle} / r_{0}\right)$ and the melting temperature $T_{\mathrm{m}}$. Thus Lindemann, long ago [32], proposed that the solid would melt when the thermal fluctuations $\sqrt{\left\langle u^{2}\right\rangle}$ exceed a given fraction (11-14\%) of the interatomic distance. More recently, Guinea et al. [33] have assimilated $T_{m}$ to the temperature at which the root mean square displacement of each atom from its equilibrium position is equal to the distance between the minimum and the inflexion point of their universal potential energy curve. This distance is roughly equal to $9 \%$ of the interatomic distance for fcc metals, which is in fair agreement with the Lindemann criterion. Such a criterion would imply that, if we plot the melting points of the eight metals in figure 4 , all these points

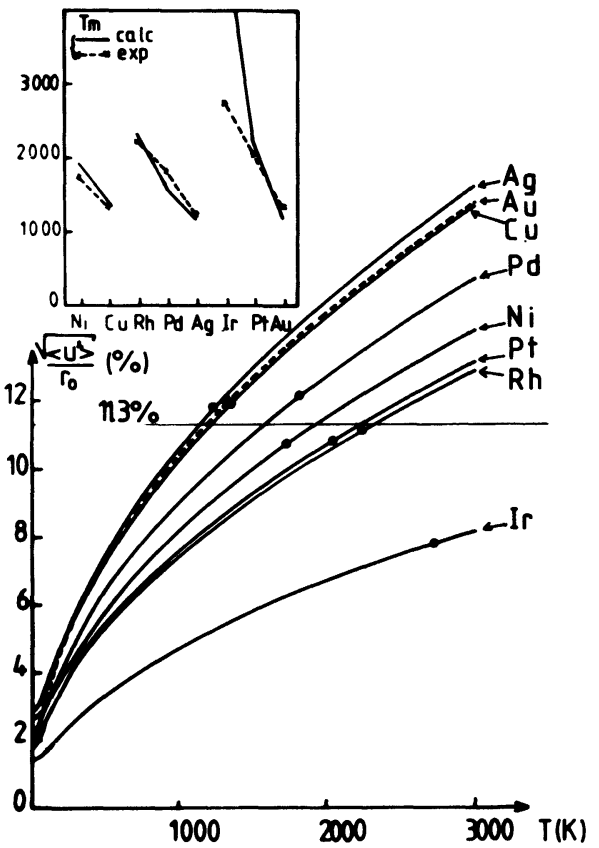

Fig. 4. - Variation of the ratio of the root mean square displacement $\left(\sqrt{\left\langle u^{2}\right\rangle}\right)$ to the interatomic spacing $\left(r_{0}\right)$ as a function of temperature. The circles indicate the experimental melting points. For the sake of clarity, the Au-curve is given in dashed line.

In the inset, we show the variation of the theoretical (-) and experimental (-----) melting temperatures along the transition series.

must be situated on the same horizontal line. One can see that this is approximately true, except for Ir; the corresponding fraction of interatomic distance is $\sim 11.3 \%$.

If we now take this ratio as the melting criterion, we can in turn evaluate theoretical melting temperatures; they are plotted and compared to experimental ones in the inset of figure 4.

Finally, from a Debye model, which is valid at high temperature, the mean square amplitude of vibration of each atom about its lattice site is [34]

$$
\left\langle u^{2}\right\rangle=\frac{9 h^{2} T}{4 \pi^{2} m k_{\mathrm{B}}\left(\theta_{\infty}^{\mathrm{B}}\right)^{2}}
$$

where $\theta_{\infty}^{\mathbf{B}}$ is the bulk Debye temperature in the high temperature limit. We can then determine $\theta_{\infty}^{\mathbf{B}}$ from the $\left\langle u^{2}\right\rangle$ curves in the high temperature range, by means of formula (15). In the other limit $(T \rightarrow 0)$, the Debye temperature $\theta_{0}^{\mathbf{B}}$ can be calculated from the energy $E$ (Eq. (14)) which at low temperature can be written

$$
E \simeq \frac{3 \pi^{4} k_{\mathrm{B}} T^{4}}{5\left(\theta_{0}^{\mathrm{B}}\right)^{3}}
$$

The Debye temperatures in both limits are plotted in figure 5 and compared with experiments. The qualitative trends are well reproduced and the quantitative 


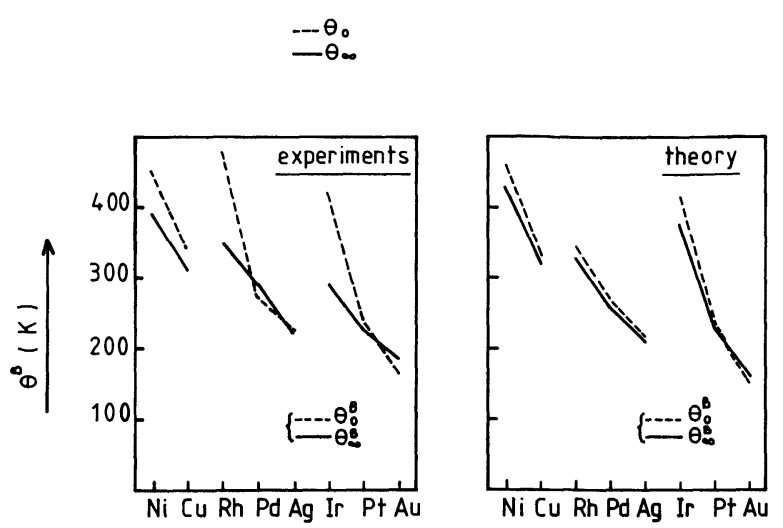

Fig. 5. - Theoretical and experimental variations of the bulk Debye temperature in the low $\left(\theta_{0}^{\mathrm{B}}:---\right)$ and high $\left(\theta_{\infty}^{\mathbf{B}}:-\right)^{-}$temperature limits along the transition series.

agreement is good except for $\mathrm{Rh}$ and Ir for which experimental data $[29,30]$ seem to indicate a large variation with $T$ which is not reproduced by our calculation. Indeed, we find very small variations of the Debye temperature between both limits, i.e. : $\theta_{0}^{\mathrm{B}} \simeq \theta_{\infty}^{\mathrm{B}} \simeq \theta_{\mathrm{D}}$.

\section{Application to low index surfaces}

Let us recall that the continued fraction technique applies without change when the periodicity of the crystal is broken by some defects, in which case it proves much more convenient than usual techniques even in the simple case of a perfect surface. Indeed, as already discussed by Black et al. [14], it allows us to work with a truly semi-infinite geometry rather than with a slab, which would lead to divergences in some numerical calculations (of $\left\langle u^{2}\right\rangle$ for instance).

In the following, we will assume that the semi-crystal retains the structure it would have as a perfect termination of the bulk metal (no relaxation or reconstruction). Moreover, we will take the same force constants at the surface as in the bulk. Let us point out that, even with such a simple assumption, the breaking of some bonds at the surface induces drastic modifications of the forces acting on surface atoms. This is true not only for the central part (broken bonds) but also for the angle bending contribution (missing triangles). Therefore, this angular contribution cannot be neglected when studying surface effects.

However, some recent experiments on Rayleigh waves of $\mathrm{Ni}(100)$ have shown that this assumption can be sometimes criticized [35]. Taking these variations into account in our formalism would set no problem and some work is currently done in the particular case of $\mathrm{Ni}$.

Let us now present our results concerning the (111), (100) and (110) surfaces of the eight fcc transition and noble metals.
5. 1 SURFACE PHONON SPECTRAL DENSITIES OF STATES. We first calculate the spectral density of states $n_{\perp}(v)$ for the motion of surface atoms perpendicular to the surface. Results are shown in figure 6 . The most striking feature is the similarity between their shapes, not only for the eight metals, but also for the three faces. More precisely, the major part of their weight is concentrated in a narrow range of low frequencies, leading to a strong peak. A more detailed analysis of these results shows that this peak is a quasi-delta function centred at about $0.38 v_{\max }$ (except for Ir : $0.48 v_{\max }$ ) for the (111) face similarly to the results of Armand [36], and it is broadened for the two others. Moreover, for the (100) face, it splits into two peaks which are due to the surface waves already found in slab calculations $[11,19]$. These surface states are centred at about $0.35 v_{\max }$ and $0.41 v_{\max }\left(0.43 v_{\max }\right.$ and $0.50 v_{\max }$ for Ir), respectively. Note that the three faces of nickel and the (100) face of rhodium have already been studied by some authors (Ni(100) [11, 12], Ni(111) [10-12], Ni(110) [13], Rh(100) [11]) using a similar continued fraction method but with a much smaller number of exact coefficients. This is the reason why neither Black et al. [11] nor Sayers [12] have been able to reproduce the splitting of surface states of $\mathrm{Ni}(100)$.

Let us define the spectral density for the motion of surface atoms parallel to the surface. Taking the perpendicular to the surface as the $z$-axis, it can be written :

$$
n_{\|}(v)=\left(n^{x}(v)+n^{y}(v)\right) / 2
$$

where the $x$ and $y$ axes are two perpendicular directions in the surface. The 24 curves are exhibited in figure 7. A glance at figure 1 shows that $n_{\|}(v)$ is more similar to the bulk density of states $(n(v))$ than $n_{\perp}(v)$. Moreover, by comparing for a given element $n_{\|}(v)$ for the three orientations one finds that the three curves are more different from one another than the corresponding $n_{\perp}(v)$. The deformation of $n_{\|}(v)$ with the orientation is qualitatively the same for all elements. This will have important consequences on the variation of $\left\langle u^{2}\right\rangle$ at the surface, as we will see below. Notice that while $n^{x}$ and $n^{y}$ are strictly degenerate for the (100) face and nearly undistinguishable for the (111), for the (110) surface, vibrations parallel and perpendicular to the closepacked $(1 \overline{1} 0)$ rows are not degenerate but show some differences, the origin of which has been discussed by Sayers [13], in the particular case of Ni (see Fig. 2 of his paper). The same behaviour has been observed for the eight fcc metals.

\subsection{VARIATION OF THE THERMODYNAMICAL PROPERTIES DUE TO THE SURFACE.}

5.2.1 Specific heat at constant volume. - It is obvious that the variation of specific heat per surface atom $\Delta C_{\mathrm{v}}$ due to the presence of a surface vanishes at $T=0 \mathrm{~K}$ and when $T$ goes to infinity. Consequently, this quantity is worth studying in the intermediate 


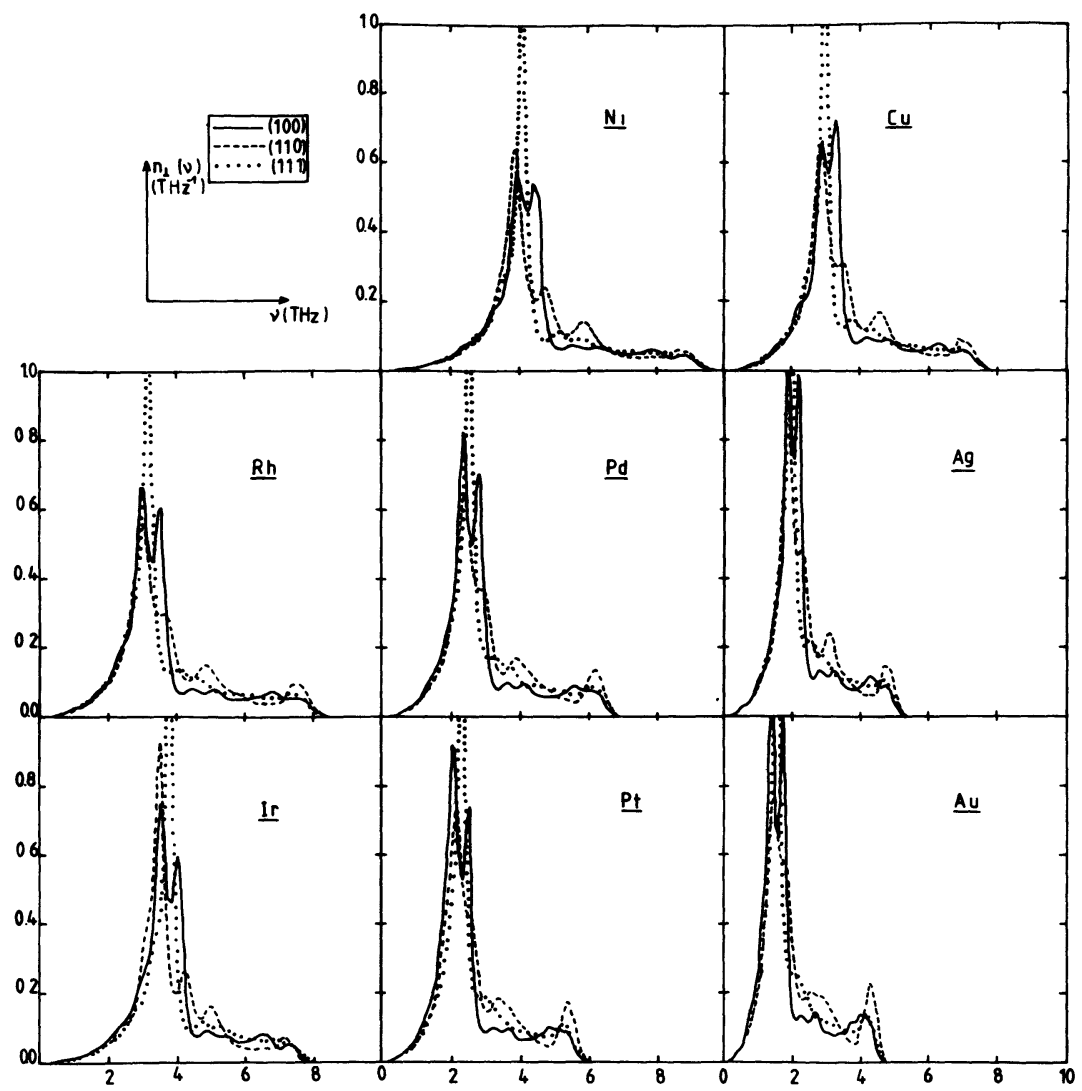

Fig. 6. - Spectral densities of states for motion of surface atoms perpendicular to the surface : $(100)$; (110); ..... (111).

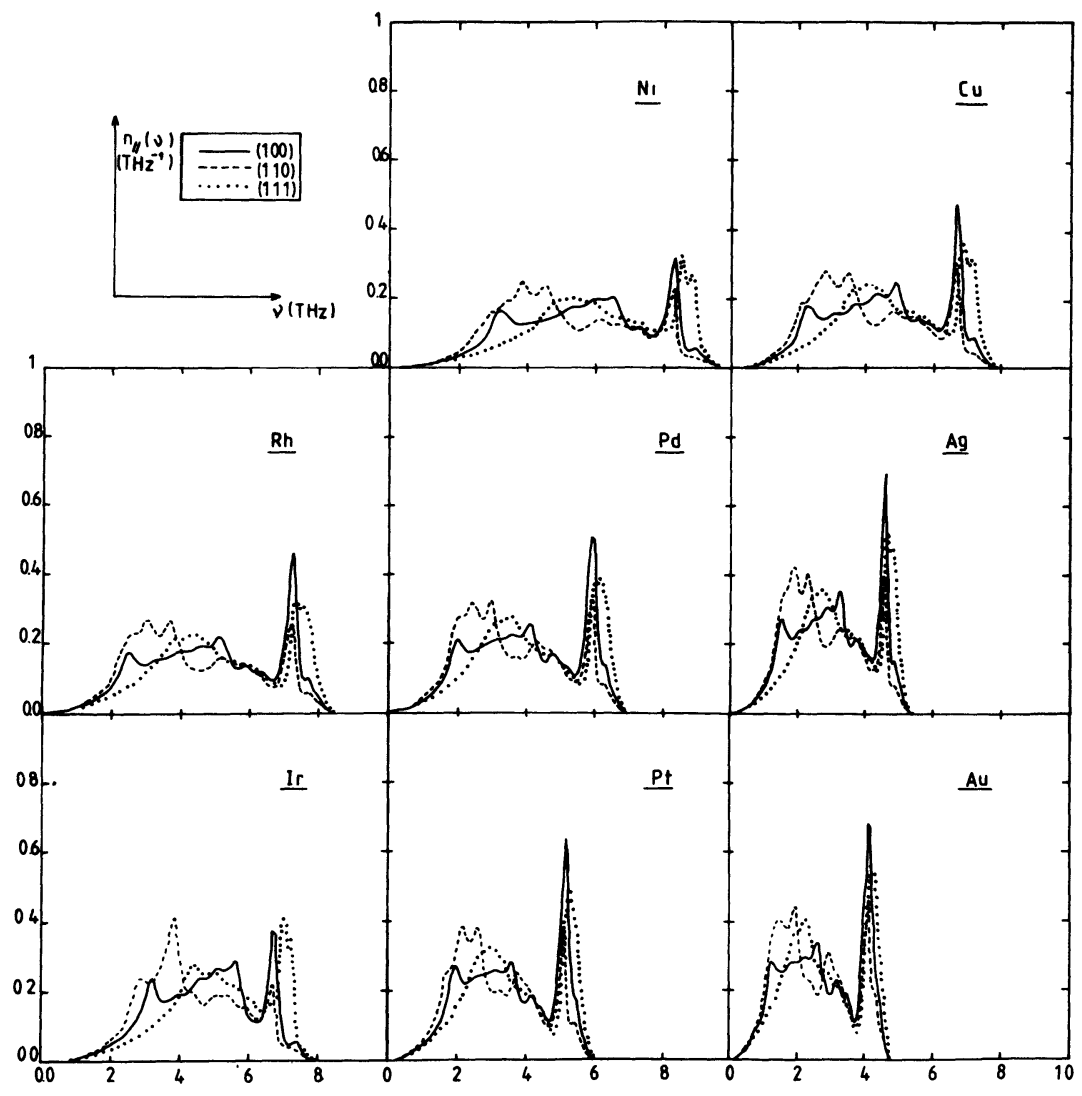

Fig. 7. - Spectral densities of states for motion of surface atoms parallel to the surface

(100), 
temperature range for which our method is particularly powerful. Indeed, in this range, Dobrzynski [3] has shown, in the particular case of the (100) face of a simple cubic crystal within the Montroll-Potts model, that $\Delta C_{\mathrm{v}}$ is always positive and presents a maximum $\Delta C_{\mathrm{v}}^{\max }$ for $T_{\max } \simeq 0.18 \theta_{\mathrm{D}}$. It is then of interest to perform a systematic study of $\Delta C_{\mathrm{v}}$ in realistic cases to see how the position and intensity of this maximum depend on the force constant model, on the element, and on the crystallographic orientation of the surface. Since we here calculate local (planar) quantities, we have to sum over their variations on successive layers :

$$
\Delta C_{\mathrm{v}}=\sum_{p=1}^{\infty} \Delta C_{\mathrm{v}}^{p}
$$

in which $\Delta C_{\mathrm{v}}^{p}$ is the contribution of the $p^{\text {th }}$ layer ( $p=1:$ surface layer)

$$
\begin{aligned}
\Delta C_{\mathrm{v}}^{p}=3 k_{\mathrm{B}} \int_{0}^{v_{\max }} & \left(n_{p}(v)-n(v)\right)\left(\frac{h v}{2 k_{\mathrm{B}} T}\right)^{2} \times \\
& \times\left(\operatorname{coth}^{2}\left(\frac{h v}{2 k_{\mathrm{B}} T}\right)-1\right) \mathrm{d} v
\end{aligned}
$$

where $n_{p}$ is the total local spectral density for a site of the $p^{\text {th }}$ layer.

In practice, $\Delta C_{\mathrm{v}}^{p}$ decreases rapidly when $p$ increases, and we will limit the summation (22) to the planes in which the atoms have not their full shell of nearest and next nearest neighbours.

Using this criterion, $\Delta C_{\mathrm{v}}^{p}$ has to be calculated for the surface layer only in the case of the (111) face and up to the second layer for the (100) and (110) faces. We have checked the accuracy of this truncation by evaluating $\Delta C_{\mathrm{v}}^{3}$ in the case of the (110) face. This contribution is indeed negligible since it is only about $2-4 \%$ of the total value. Let us notice however that the contribution of the second layer in this case is significant ( $\sim 20 \%$ of the total value). The results are shown in figure 8 . Obviously, the value of $\Delta C_{\mathrm{v}}^{\max }$ increases with the number of broken bonds at the surface (inset of Fig. 8). More surprisingly the corresponding temperature $T_{\max }$ is roughly independent of the face. If we now scale $T_{\max }$ by the Debye temperature of the metal of interest, one finds that this ratio is almost the same for all metals.

$$
\frac{T_{\max }}{\theta_{\mathrm{D}}} \simeq 0.175
$$

which generalizes the prediction of Dobrzynski [3] to the case of all low index faces and of all fcc transition and noble metals.

5.2.2 Vibrational entropy. - Similarly to $\Delta C_{v}$, the variation of entropy per surface atom can be written

$$
\Delta S=\sum_{p=1, p_{\mathrm{m}}} \Delta S^{p}
$$

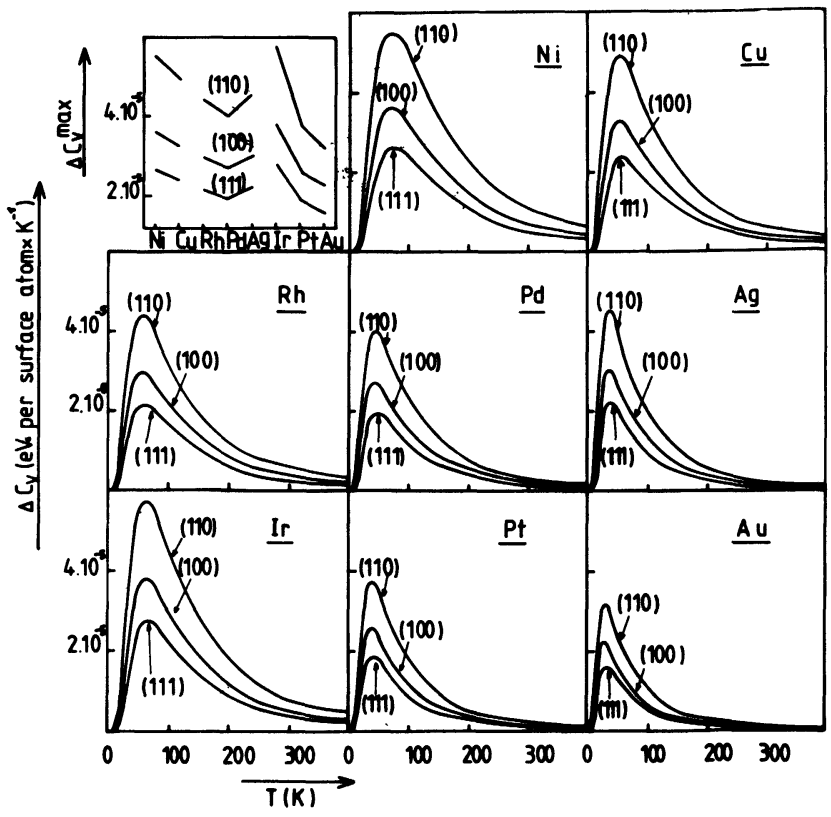

Fig. 8. - Variation of specific heat $\Delta C_{\mathrm{y}}$ due to the presence of the surface, for the three low index faces, as a function of temperature. In the inset is shown the evolution of the maximum value of $\Delta C_{\mathrm{v}}$ along the transition series.

with $p_{\mathrm{m}}=1$ for the (111) face and $p_{\mathrm{m}}=2$ for the two others. The corresponding curves (Fig. 9) have a well known shape [3] : they increase from zero at $T=0 \mathrm{~K}$ and tend to a constant value when $T$ goes to infinity. Obviously, this constant value increases with the number of broken bonds. The order of magnitude of $\Delta S$ (a few $\left.10^{-1} \mathrm{erg} / \mathrm{cm}^{2} / \mathrm{K}\right)$ is consistent with expe-

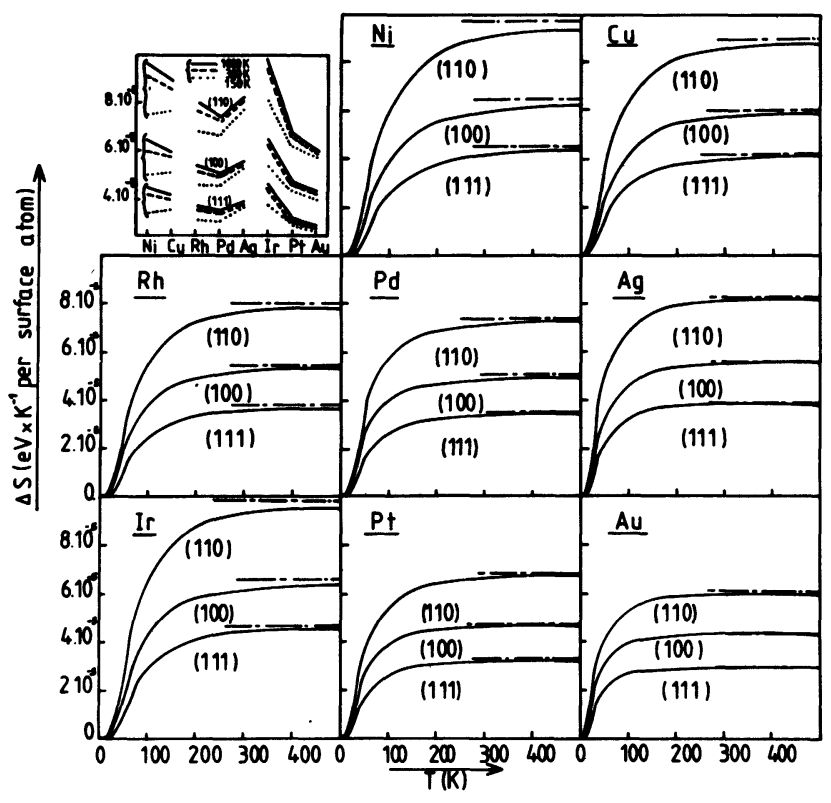

Fig. 9. - Variation of the entropy $\Delta S$ due to the presence of the surface for the three low index faces as a function of temperature. In the inset, we show the evolution of $\Delta S$ along the transition series at given temperatures. 
rimental data [37]. However, in view of the experimental uncertainties, a detailed comparison would not be meaningful. Finally, let us notice that our results are in good agreement with those of DjafariRouhani et al. [6], calculated for various models in the high temperature limit.

5.2.3 Surface mean square displacements. - From the spectral densities of states $n_{\perp}(v)$ and $n_{\|}(v)$, it is easy to calculate (Eq. (15)) the root mean square displacements $\sqrt{\left\langle u^{2}\right\rangle_{\perp}^{s}}$ and $\sqrt{\left\langle u^{2}\right\rangle_{\|}^{s}}$ for surface atoms. Let us consider the ratio of these quantities to the values they would have in the bulk $: \sqrt{\left\langle u^{2}\right\rangle^{\mathbf{B}}}$ :

$$
r_{\alpha}^{s}=\sqrt{\frac{\left\langle u^{2}\right\rangle_{\alpha}^{s}}{\left\langle u^{2}\right\rangle^{\mathrm{B}}}}, \quad \alpha=\perp \|
$$

This ratio obviously tends to a constant value at high temperature. We have performed here the calculation in the whole range of temperature. The results are given at $T=600 \mathrm{~K}$ in figure 10a but all the qualitative trends that we can derive from this figure are valid for any temperature. As expected, the root mean square displacements which are perpendicular to the surface undergo the greatest change with respect to the bulk value. On the other hand, the ratio $r_{\alpha}^{s}$ is nearly the same for all metals. Moreover, $r_{\perp}^{s}$ is almost independent of the surface orientation, a good average value of this ratio would be $r_{\perp}^{s} \simeq 1.40$ at high temperature. On the contrary, $r_{\|}^{s}$ depends significantly on this surface orientation : the three average values in the high temperature limit should be 1.10, 1.18 and 1.27 for the (111), (100), and (110) faces respectively. This confirms the conjecture by Goodman et al. [38] based on experimental data on Pd [38] and Pt [39], that the parallel displacements might show a more pronounced dependence on the surface density of atoms than the perpendicular one (similarly to the spectral densities $n_{\|}(v)$ and $n_{\perp}(v)$ ). If we now consider the intermediate range of temperature, the ratios $r_{\alpha}^{s}$ can be
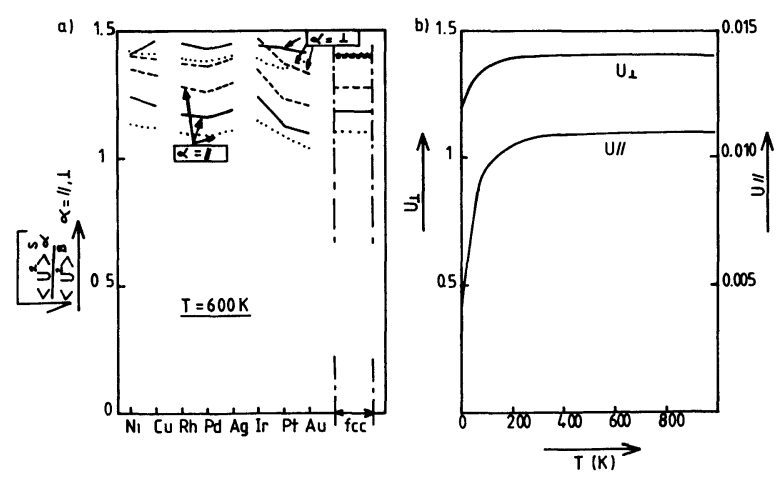

Fig. 10. - (a) Variation of the ratios $r_{\alpha}^{\mathrm{s}}$ (see text); $\alpha=\perp$, $\|$, at a given temperature $(T=600 \mathrm{~K})$ along the transition series. $-(100),----(110) ; \ldots . . .(111)$.

The resulting mean values for fcc metals are given on the right hand side. (b) Variation of the parameters $U_{\perp}$ and $U_{\|}$ defined in equation (26) as a function of temperature. represented to a good accuracy, by the following simple formulae

$$
\begin{aligned}
& r_{\perp}^{s}=U_{\perp}(T) \\
& r_{\|}^{s}=1+U_{\|}(T) \cdot \delta p_{\mathrm{s}}^{2}
\end{aligned}
$$

where $\delta p_{\mathrm{s}}$ is the number of missing bonds of surface atoms for a given surface orientation $\mathrm{s}$ and $U_{\perp}$ and $U_{\|}$ are shown in figure $10 \mathrm{~b}$ as a function of temperature. One can consider that these functions have reached their asymptotic values $\left(U_{\perp}=1.40\right.$ and $\left.U_{\|}=0.011\right)$ for $T>300 \mathrm{~K}$. Note that the ratio $\left(r_{\perp}^{s} / r_{\|}^{s}\right)$, which is a measure of the anisotropy of the root mean square displacement, is closest to 1 for the (110) surface.

As a conclusion we have found that the surface vibrational amplitudes normal to the surface plane are about $40 \%$ larger than the corresponding bulk values for the three low index faces of the eight fcc transition metals. Some experimental data $[38,39]$ indicate that this increase should be between $40 \%-100 \%$. Therefore, our calculation seems to underestimate this variation. This can be due to possible variations of force constants near the surface or to anharmonic corrections [40] which are neglected here.

From the ratio of the surface and bulk components of mean square displacements, one can calculate the ratio of the squares of the surface and bulk Debye temperatures using equation (19) :

$$
\frac{\left\langle u^{2}\right\rangle^{\mathrm{s}}}{\left\langle u^{2}\right\rangle^{\mathrm{B}}}=\left(\frac{\theta_{\infty}^{\mathrm{B}}}{\theta_{\infty}^{\mathrm{s}}}\right)^{2}
$$

Using equation (27) with the total mean square displacement, one obtains an averaged surface Debye temperature which is compared to the bulk temperature in figure 11. However, experimental data are only available for the perpendicular component ratio. A good approximation of the surface Debye temperature associated with displacements perpendicular

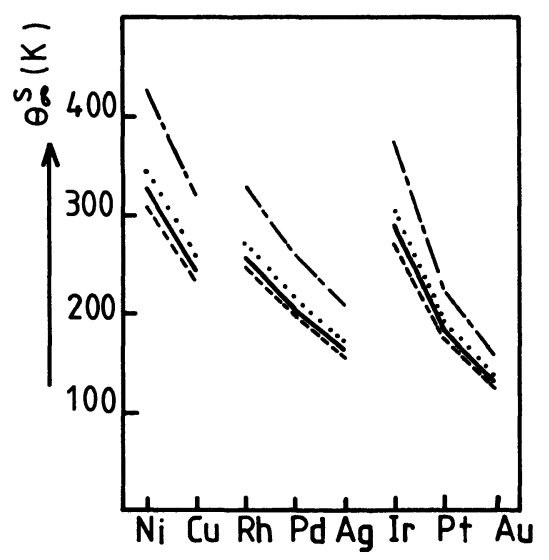

Fig. 11. - Variation of the averaged surface Debye temperature $\left(\theta_{\infty}^{\mathrm{s}}\right)$ in the high temperature limit along the transition series $\{-(100)$, ----- (110); ..... (111) $\}$ compared to the bulk one $\left(\theta_{\infty}^{\mathrm{B}}-\cdot-\cdot\right)$. 
to the surface can be obtained from formula (26), i.e. :

$$
\theta_{\infty, \perp}^{\mathrm{s}} \simeq 0.7 \theta_{\infty}^{\mathrm{B}} \text {. }
$$

Then $\theta_{\infty, 1}^{\mathrm{s}}$ should be independent of the surface orientation; this is indeed confirmed by experiments performed in particular on the (100) and (111) faces of $\mathrm{Rh}$ [15] and Pd [38] and on the three low index faces of Pt [39]. The experimental ratios $\left(\theta_{\infty, 1}^{\mathbf{s}} / \theta_{\infty}^{\mathbf{B}}\right)$ are found between 0.5-0.7 for $\mathrm{Pt}, \mathrm{Pd}, \mathrm{Rh}$ and $\mathrm{Ag}$. Comparison between these experimental data and equation (28) shows that the calculated values are, on the average, slightly overestimated : this is clearly due to the underestimation of $\left\langle u^{2}\right\rangle_{\perp}^{\mathbf{s}}$ at the surface previously discussed.

Finally let us briefly comment on the melting at surfaces. A naive approximation would be to use the Lindemann criterion with the same critical ratio $\frac{\sqrt{\left\langle u^{2}\right\rangle}}{r_{0}}=11.3 \%$, as in the bulk. Since the mean square displacements are larger for surface atoms than for bulk atoms, this criterion leads to a surface melting temperature lower than the bulk one [41] (see Table II). Moreover, the order of melting temperatures corresponds to the intuitive picture that a loose-packed surface melts before a close-packed one. In addition, table II seems to indicate that the differences between surface and bulk melting temperatures might decrease from the first to the third transition series. However, anharmonic effects are known to be non-negligible near the melting temperature and could be sensitive to the surface. Furthermore it has been shown that taking relaxation effects into account could reverse the order of melting temperatures [42].

\section{Conclusion}

To summarize, we have shown that the continued fraction method, which is known to be a very powerful technique for the study of the electronic structure of transition metals, can also be fruitfully applied to investigate vibrational and thermodynamical properties. Actually, it only requires very short computation time even when the crystal symmetry is broken by defects. In addition, the calculation can be performed to any degree of accuracy by adjusting the number of exact levels in the continued fraction (i.e. varying the cluster size). Consequently, systematic studies can be undertaken such as that presented here. Our results on bulk fcc transition and noble metals reproduce all the experimental trends fairly well; the quantitative agreement is also quite good except for some properties of Ir. Let us however recall that in the last case no experimental phonon dispersion curves seem to be available; the knowledge of such curves would allow us to refine our force constants. The
Table II. - Variation of the ratio $\frac{T_{\mathrm{m}}^{\mathrm{S}}}{T_{\mathrm{m}}^{\mathrm{B}}}\left(T_{\mathrm{m}}^{\mathrm{S}(\mathrm{B})}:\right.$ surface (bulk) melting temperature calculated using the same Lindemann criterion) with the surface orientation $\{S=(110),(100),(111)\}$ and the nature of the elements (1st, 2 nd and 3 rd transition series).

\begin{tabular}{|c|c|c|c|}
\cline { 2 - 4 } \multicolumn{1}{c|}{} & fcc : 3d-series & fcc $:$ 4d-series & fcc $:$ 5d-series \\
\hline$T_{\mathrm{m}}^{(110)} / T_{\mathrm{m}}^{\mathrm{B}}$ & 0.72 & 0.77 & 0.83 \\
\hline$T_{\mathrm{m}}^{(100)} / T_{\mathrm{m}}^{\mathrm{B}}$ & 0.78 & 0.83 & 0.90 \\
\hline$T_{\mathrm{m}}^{(111)} / T_{\mathrm{m}}^{\mathrm{B}}$ & 0.87 & 0.92 & 0.97 \\
\hline
\end{tabular}

natural continuation of our study would be to extend the systematics to bcc and hcp metals.

In the case of surfaces, we have put forward some fairly general trends. In particular, the variation of the specific heat at constant volume per surface atom is maximum at a temperature which is a constant fraction $(\sim 0.175)$ of the bulk Debye temperature, for any metal and surface orientation. On the other hand, we have found that the lateral displacement of surface atoms shows a more pronounced dependence on the surface density of atoms than the perpendicular one. This trend is confirmed by existing experiments. However, from a quantitative point of view, our calculations seem to underestimate the difference between surface and bulk atoms. Further studies on surfaces should take into account the possible variation of force constants near the surfaces which might be due, for instance, to relaxation or reconstruction phenomena.

Comments. - One of the referees has drawn our attention to the work of Jayanthi et al. [43] which has been published after this manuscript was sent for publication. These authors use a self-consistent harmonic method to study the thermal properties of $\mathrm{Cu}$ with a pairwise central short ranged potential. It is therefore interesting to compare our results to theirs since, even though angular forces have been taken into account in our work, their influence is small in $\mathrm{Cu}$. In view of this comparison (particularly the adequacy of the Lindemann criterion for melting), it appears that, at least for $\mathrm{Cu}$, anharmonic effects are not of prime importance.

\section{Acknowledgments.}

We are indebted to Drs. F. Ducastelle, D. Spanjaard, B. Legrand and P. Turchi for many fruitful discussions and a critical reading of the manuscript. 


\section{Appendix.}

CALCULATION OF THE DYNAMICAL MATRIX ELEMENTS. - The dynamical matrix elements are given by :

$$
\begin{aligned}
& \mathbb{D}_{00}^{\lambda \mu}=\frac{1}{4 \pi^{2} m}\left(\sum_{\{0, i\}}\left\{\lambda_{i} \delta^{\lambda \mu}+\left(\beta_{i}-\lambda_{i}\right) d_{0 i}^{\lambda} d_{0 i}^{\mu}\right\}+\sum_{\{0, i, j\}}\left\{\left(Q_{0 i}+Q_{0 j}\right) \delta^{\lambda \mu}+P_{0 i}^{\lambda \mu}+P_{0 j}^{\lambda \mu}\right\}\right) \\
& \mathbb{D}_{0 i}^{\lambda \mu}=\frac{1}{4 \pi^{2} m}\left(-\lambda_{i} \delta^{\lambda \mu}-\left(\beta_{i}-\lambda_{i}\right) d_{0 i}^{\lambda} d_{0 i}^{\mu}+\sum_{\{0, j\}}\left\{\left(Q_{i j}-Q_{0 i}\right) \delta^{\lambda \mu}+P_{i j}^{\lambda \mu}-P_{0 i}^{\lambda \mu}\right\}\right)
\end{aligned}
$$

The notations have been defined in the main text. There remains to give the explicit expressions of $P$ and $Q$ :

$$
\begin{aligned}
& P_{0 i}^{\lambda \mu}=x A_{0 i} d_{0 i}^{\lambda} d_{0 i}^{\mu}+x B_{0 i} d_{0 j}^{\lambda} d_{0 i}^{\mu}+x^{2} C_{0 i}\left(d_{i j}^{\lambda} d_{0 i}^{\mu}+d_{0 i}^{\lambda} d_{i j}^{\mu}\right)+x D_{0 i}^{0 j} d_{0 i}^{\lambda} d_{0 j}^{\mu}+x E_{0 i}^{0 j} d_{0 j}^{\lambda} d_{0 j}^{\mu}+x^{2} F_{0 i}^{i j} d_{i j}^{\lambda} d_{i j}^{\mu} \\
& P_{0 j}^{\lambda \mu}=A_{0 j} d_{0 j}^{\lambda} d_{0 j}^{\mu}+B_{0 j} d_{0 i}^{\lambda} d_{0 j}^{\mu}+C_{0 j}\left(d_{i j}^{\lambda} d_{0 j}^{\mu}+d_{0 j}^{\lambda} d_{i j}^{\mu}\right)+D_{0 i}^{0 j} d_{0 j}^{\lambda} d_{0 i}^{\mu}+E_{0 i}^{0 j} d_{0 i}^{\lambda} d_{0 i}^{\mu}+F_{0 j}^{i j} d_{i j}^{\lambda} d_{i j}^{\mu} \\
& P_{i j}^{\lambda \mu}=x y A_{i j} d_{0 i}^{\lambda} d_{i j}^{\mu}+y B_{i j} d_{0 j}^{\lambda} d_{i j}^{\mu}+y C_{i j} d_{i j}^{\lambda} d_{i j}^{\mu}+x y C_{0 i} d_{0 i}^{\lambda} d_{0 i}^{\mu}+y C_{0 j} d_{0 j}^{\lambda} d_{0 j}^{\mu}+x y F_{0 i}^{i j} d_{i j}^{\lambda} d_{0 i}^{\mu}+y F_{0 j}^{i j} d_{i j}^{\lambda} d_{0 j}^{\mu} \\
& \text { where }\left|\mathbf{R}_{0 j}\right|=r ;\left|\mathbf{R}_{0 i}\right|=\frac{r}{x} ;\left|\mathbf{R}_{i j}\right|=\frac{r}{y} \text {. } \\
& \text { and } \\
& \begin{array}{l}
Q_{0 i}=\Gamma_{0}\left(1-x \cos \theta_{0}\right) x-\Gamma_{i} x^{2} \cos \theta_{i} \\
Q_{0 j}=\Gamma_{0}\left(x-\cos \theta_{0}\right)-\Gamma_{j} \cos \theta_{j} \\
Q_{i j}=y\left(\Gamma_{j}-x \Gamma_{i}\right) \\
A_{0 i}=\Gamma_{0} x^{3}\left(3 x \cos \theta_{0}-1\right)+\Delta_{0} x^{3}\left(x \cos ^{2} \theta_{0}-\cos \theta_{0}\right)+x^{4} \cos \theta_{i}\left(3 \Gamma_{i}+\Delta_{i} \cos \theta_{i}\right) \\
A_{0 j}=\Gamma_{0}\left(3 \cos \theta_{0}-x\right)+\Delta_{0}\left(\cos ^{2} \theta_{0}-x \cos \theta_{0}\right)+\cos \theta_{j}\left(3 \Gamma_{j}+\Delta_{j} \cos \theta_{j}\right) \\
A_{i j}=x^{2} y^{2} \cos \theta_{i}\left(\Gamma_{i}+\Delta_{i} \cos \theta_{i}\right) \\
B_{0 i}=x^{2}\left(\cos \theta_{0}-x\right)\left(\Gamma_{0}+\Delta_{0} \cos \theta_{0}\right) \\
B_{0 j}=x\left(x \cos \theta_{0}-1\right)\left(\Gamma_{0}+\Delta_{0} \cos \theta_{0}\right) \\
B_{i j}=y^{2} \cos \theta_{j}\left(\Gamma_{j}+\Delta_{j} \cos \theta_{j}\right) \\
C_{0 i}=y x^{3}\left(\Gamma_{i}+\Delta_{i} \cos \theta_{i}\right) \\
C_{0 j}=-y\left(\Gamma_{j}+\Delta_{j} \cos \theta_{j}\right) \\
C_{i j}=x y^{3}\left(\Gamma_{i}+\Delta_{i} \cos \theta_{i}\right)-y^{3}\left(\Gamma_{j}+\Delta_{j} \cos \theta_{j}\right) \\
D_{0 i}^{0 j}=x^{2}\left(-x \Gamma_{0}+\Delta_{0}\left(1-x \cos \theta_{0}\right)\right) \\
E_{0 i}^{0 j}=x\left(-\Gamma_{0}+\Delta_{0}\left(x-\cos \theta_{0}\right)\right) \\
F_{0 i}^{i j}=x^{2} y^{2} \Delta_{i} \\
F_{0 j}^{i j}=y^{2} \Delta_{j}
\end{array}
\end{aligned}
$$

with :

$$
\begin{aligned}
& \theta_{0}=\theta_{i \hat{0} j} ; \quad \theta_{i}=\theta_{0 \hat{i} j} \quad \text { and } \quad \theta_{j}=\theta_{0 \hat{j} i} \\
& \Gamma_{0}=\frac{\gamma_{i 0 j}}{r^{2}} ; \quad \Gamma_{i}=\frac{\gamma_{0 i j}}{r^{2}} \text { and } \quad \Gamma_{j}=\frac{\gamma_{0 j i}}{r^{2}}
\end{aligned}
$$

and the same for $\Delta_{0}, \Delta_{i}$ and $\Delta_{j}$.

Finally, let us notice that in the particular case of fcc crystals, these expressions can be written in a much simpler way since all triangles are equilateral so that

$$
\begin{aligned}
x & =y=1 \\
\theta_{0} & =\theta_{i}=\theta_{j}=\frac{\pi}{3} \\
\Gamma_{0} & =\Gamma_{i}=\Gamma_{j}=\Delta_{0}=\Delta_{i}=\Delta_{j} .
\end{aligned}
$$




\section{References}

[1] Maradudin, A. A., Montroll, E. M., Weiss, G. H. and Ipatova, I. P., in Solid State Phys. Suppl. 3 (1971) (Academic Press-New York).

[2] Maradudin, A. A., Wallis, R. F. and Dobrzynski, L., Handbook of Surfaces and Interfaces 3 (1980) (Garland STPM Press, New York and London).

[3] Dobrzynski, L., Ann. Phys. 4 (1969) 637.

[4] Dobrzynski, L. and Friedel, J., Surf. Sci. 12 (1968) 469.

[5] Friedel, J., J. Physique Lett. 35 (1974) L-59.

[6] DJafari-Rouhani, B., Dobrzynski, L. and Allan, G., Surf. Sci. 55 (1976) 663.

MASRI, P. and DoBRZYNSKI, L., Surf. Sci. 32 (1972) 623.

[7] Moraitis, G. and Gautier, F., J. Phys. F 7 (1977) 1421.

[8] Jelitto, R. J., J. Phys. Chem. Solids 30 (1969) 609.

[9] Kelly, M. J., Solid State Phys. 35 (1980) 356.

[10] BlaCK, J. E., Surf. Sci. 105 (1981) 59.

[11] Black, J. E., Campbell, D. A. and Wallis, R. F., Surf. Sci. 105 (1981) 629.

[12] SAYers, C. M., J. Phys. C 16 (1983) 2381.

[13] SAYers, C. M., Surf. Sci. 136 (1984) 582.

[14] Black, J. E., Laks, B. and Mills, D. L., Phys. Rev. B 22 (1980) 1818

[15] Castner, D. G., Somorjai, G. A., Black, J. E., CasTIEL, D. and Wallis, R. F., Phys. Rev. B 24 (1981) 1616.

[16] Hersovici, C. and Fibich, M., J. Phys. C 13 (1980) 1635.

[17] MeeK, P. E., Philos. Mag. 33 (1976) 897.

[18] Hashimoto, M., Ishida, Y., Yamamoto, R. and Doyama, M., Acta Met. 29 (1981) 617.

[19] Castiel, D., Dobrzynski, L. and SPanjaARd, D., Surf. Sci. 59 (1976) 252.

[20] Haydock, R., Heine, V. and Kelly, M. J., J. Phys. C 5 (1972) 2845.

[21] Gaspard, J. P. and Cyrot-Lackmann, F., J. Phys. C 6 (1973) 3077.

[22] Birgeneau, R. J., Cordes, J., Doling, G. and Woods, A. D. B., Phys. Rev. 136 (1964) 1359.

[23] Nicklow, R. M., Gilat, G., Smith, H. G., RaubenheIMER, L. J. and Wilkinson, M. K., Phys. Rev. 164 (1967) 922.

[24] Milller, A. P. and Brockhouse, B. N., Can. J. Phys. 49 (1971) 704
[25] Kamitakahara, W. A. and Brockhouse, B. N., Phys. Lett. 29A (1969) 639.

[26] Dutton, D. H. and Brockhouse, B. N., Can. J. Phys. 50 (1972) 2915.

[27] LynN, J. W., Smith, H. G. and Nicklow, R. M., Phys. Rev. B 8 (1973) 3493.

[28] Ducastelle, F., J. Physique 31 (1970) 1055.

[29] American Institute of Physics Handbook (IIIrd edition) D. E. Gray editor (McGraw Hill Co) 1972, p. 4-115.

[30] White, G. K., Experimental techniques in low-temperature physics (Clarendon Press Oxford) 1968, p. 318.

[31] Hultgren, R., OrR, R. L., Anderson, P. D. and KELLEY, K. K., Selected values of thermodynamic properties of metals and alloys (John Wiley and Sons, inc.) 1963.

[32] Lindemann, F. A., Z. Phys. 11 (1910) 609.

[33] Guinea, F., Rose, J. H., Smith, J. R. and Ferrante, J., Appl. Phys. Lett. 44 (1984) 53.

[34] Ziman, J. M., Principles of the theory of solids (Cambridge Univ. Press) 1964, p. 62.

[35] Szeftel, J. and Lehwald, S., Surf. Sci. 143 (1984) 11.

[36] Armand, G., Solid State Commun. 48 (1983) 261.

[37] Funk, E. R., Udin, H. and WulfF, J., J. Metals 3 (1951) 1206.

ButTNER, F. H., Udin, H. and WulfF, J., J. Metals 3 (1951) 1209.

Udin, H., Shaler, A. J. and WulfF, J., J. Metals 1 (1949) 1936.

[38] Goodman, R. M., Farrell, H. H. and Somorjai, G. A., J. Chem. Phys. 48 (1968) 1046.

[39] Lyon, H. B. and Somorjai, G. A., J. Chem. Phys. 44 (1966) 3707

[40] Clark, B. C., Herman, R. and Wallis, R. F., Phys. Rev. 139 (1965) 860.

[41] Pietronero, L. and Tosatti, E., Solid State Commun. 32 (1979) 255.

[42] Jayanthi, C. S. and Tosatti, E., Proceedings of the 6th European Conference on Surface Science (York), European Conference abstracts Vol. 8B (1984) 35.

[43] Jayanthi, C. S., Tosatti, E. and Fasolino, A., Phys. Rev. B 31 (1985) 470. 